SLAC-PUB-6239

SLAC/SSRL-0032

May 1993

(SSRL-M)

\title{
ATOMIC AND ELECTRONIC STRUCTURE OF B/SI(100)*
}

\author{
R. Cao, X. Yang, and P. Pianetta \\ Stanford Linear Accelerator Center, Stanford Synchrotron Radiation Laboratory \\ Stanford, CA 94309, U.S.A.
}

\begin{abstract}
The atomic and electronic structures of the $\mathrm{B} / \mathrm{Si}(100)$ surface has been studied using high resolution core level and valence band photoelectron spectroscopy as well as low energy electron diffraction. The structure models for such a surface have been proposed, which consist of boron atoms residing on the top surface adatom sites. Experimental evidence shows that this surface is not a dangling bond free surface. Epitaxial growth of Si and Ge on this structure has been achieved at relatively low temperature, and our results indicate that boron atoms underneath the epitaxial layer act as acceptors. As a result, conversion of n-type to p-type region takes place near the surface. Comparison of this surface with the other group-III metals on $\mathrm{Si}(100)$ surface has also been conducted.
\end{abstract}

Journal of Vacuum Science and Technology B

* Work Supported by the U.S. Department of Energy under Contract DE-AC03-76SF00515 


\section{Introduction}

$\mathrm{B} / \mathrm{Si}$ surface is of interest because $\mathrm{B}$ on $\mathrm{Si}$ surfaces often shows unique structures compared with other well understood group-III metals. For instance, extensive studies have shown that all the group-III metals on $\operatorname{Si}(111)$ can form $(\sqrt{3} \times \sqrt{3})$ reconstruction, but B distinguishes itself from the others by the capability of occupying the subsurface sites [13]. The unique $\mathrm{B} / \mathrm{Si}(111)$ surface also shows different electronic properties and may lead

to interesting applications. Passivation of the $\mathrm{Si}$ surface and atomic scale negative differential resistance are just the few of the many possible applications of its unique structure [4,5]. An analogous situation exists for the group-III metals on $\mathrm{Si}(100)$ surface. It has been reported that $\mathrm{Al}, \mathrm{Ga}$, and In can show $(2 \times 2)$ reconstruction at a coverage of $1 / 2$ monolayer $(\mathrm{ML})[6]$. In contrast, $\mathrm{B} / \mathrm{S}(100)$ exhibits a $(2 \times 1)$ reconstruction at the same coverage [7]. More interestingly, $\mathrm{B} / \mathrm{Si}(100)$ shows great potential in device application. This mainly comes from the recent finding that one is able to achieve Si epitaxial growth on this structure at relatively low temperature and the B layer underneath the epitaxial layer behaves like a delta doping layer. So far, most studies have been confined on the process of formation of ordered B layer and epitaxial growth afterwards on the $\mathrm{Si}(100)$ surface. Little has been understood regarding the structural and electronic properties of this surface at atomic scale. This is exactly the goal of current work, to characterize the atomic and electronic properties of this unique and important structure.

\section{Experimental}

The experiments were conducted at the Stanford Synchrotron Radiation Laboratory (SSRL) on both beam line III-1 with a grasshopper monochromator and beam line VIII-1 with a toroidal grating monochromator. A VG ADES 400 angle resolved photoemission ultra high vacuum (UHV) chamber equipped with a hemispherical spectrometer and an angle integrated photoemission system with a double-pass cylindrical analyzer (CMA) were 
utilized. The combined energy resolution was less than $0.25 \mathrm{eV}$ for the angle resolved system with angle resolution about $2^{\circ}$. The surface reconstruction was characterized by an in situ LEED optics. The n-type Si(100) wafers were cleaned before inserted into the UHV systems with a base pressure around $2 \times 10^{-10}$ torr. They were outgassed at $\sim 600^{\circ} \mathrm{C}$ and at low pressure for few hours. The clean surfaces were finally generated by heating the wafers up to $1000^{\circ} \mathrm{C}$ for short period of time. Sharp two domain $(2 \times 1)$ LEED patterns were observed afterwards. $\mathrm{B} / \mathrm{Si}(100)$ surface was prepared through evaporating solid $\mathrm{B}_{2} \mathrm{O}_{3}$ mounted on a tungsten filament while the Si wafers were held at about $500^{\circ} \mathrm{C}$ during deposition. The amount of the deposited material was determined by the evaporation rate calibrated from an in situ crystal thickness monitor. This surface was then annealed at a temperature higher than $700^{\circ} \mathrm{C}$. The photoemission spectra and LEED show that the post annealing drives oxygen away from the system and yields a sharp (two domain) $(2 \times 1)$ surface reconstruction. Epitaxial growth of Si and Ge layer on top of the B/Si(100) structure with or without $\mathrm{Sb}$ surfactant assistance was conducted in the same system near the molecular beam epitaxy (MBE) growth condition using solid Si and Ge sources. The band bending and the surface Fermi level movement upon $\mathrm{B} / \mathrm{Si}(100)$ formation and subsequent epitaxial growth were determined by following the bulk component of the Si $2 p$ core level spectra.

\section{Results and Discussions}

Figure 1 illustrates the process of formation of the $\mathrm{B} / \mathrm{Si}(100)$ surface. Here the $\mathrm{B} 1 \mathrm{~s}$ core level spectra $(\mathrm{hv}=220 \mathrm{eV})$ of $\mathrm{B}$ on the $\mathrm{Si}(100)$ surface as a function of annealing temperature are shown. The top spectrum was taken when $\mathrm{B}_{2} \mathrm{O}_{3}$ was deposited on the $\mathrm{Si}(100)$ surface held at $500^{\circ} \mathrm{C}$. The spectrum contains two peaks. The main peak located at about $26.5 \mathrm{eV}$ kinetic energy comes from the B atoms bound to oxygen atoms, indicating that the species on the surface upon deposition is mainly in a form of $\mathrm{B}_{2} \mathrm{O}_{3}$ molecule. A 
small component on the low bind energy side may result from break-up of some B-O bonds in the $\mathrm{B}_{2} \mathrm{O}_{3}$ molecules. A diffused $(1 \times 1)$ LEED pattern was observed upon $\mathrm{B}_{2} \mathrm{O}_{3}$ deposition, indicating that the Si surface was covered by a disordered layer. After $750^{\circ} \mathrm{C}$ annealing, a rather large shift of the $\mathrm{B} 1 \mathrm{~s}$ peak about $5 \mathrm{eV}$ towards the lower binding energy takes place. At the same time, all the photoemission spectra show no indication of presence of any form of oxygen on the surface. Meanwhile, a sharp $(2 \times 1)$ LEED pattern is observed. These results essentially suggest a complete break of the B-O bonds, desorption of oxygen from the surface, and full incorporation of $\mathrm{B}$ atoms with the Si substrate, in agreement with a recent report by Weir et al $[7,8]$. The large shift of the B 1 s core level position is due to the fact that boron tends to give away charges when bounded to oxygen while takes charges when bounded to silicon, using a simple electronegativity argument $(3.44,2.04$, and 1.90 on Pauling scale for O, B, and Si, respectively). As the annealing temperature further increases, the B 1s peak remains at roughly the same position bat the intensity starts to decrease. The reduction of intensity is attributed to diffusion of the surface boron atoms into the substrate.

The Si $2 p$ core level spectra of the clean and B covered $\mathrm{Si}(100)$ surfaces are presented in figure 2. In order to illustrate the evolution of the surface, all the spectra have been decomposed into different components using a computer least square fitting. The fitting parameters are approximately the same as those we have used before [9]. The clean $\operatorname{Si}(100)-(2 \times 1)$ spectrum consists of three components. It has been established that these components represent the contribution from the bulk (B), the surface dimers (S), and the sublayer Si atoms $\left(S^{\prime}\right)$, respectively $[9,10]$. After B deposition followed by a $750^{\circ} \mathrm{C}$ annealing, the original surface component on the lower binding energy side disappears. Instead, a new chemically shifted component, $S^{*}$, on the high binding energy side shows up. Rest of the spectrum is, to large extent, similar to that of the original surface. This new component is assigned as a B-related Si component. Again, shifting of this -4 - 
component towards high binding energy direction is consistent with the fact that $\mathrm{B}$ is more electronegative than the Si counterpart so that charge is drawn from Si to B. As stated before, in this case the surface already exhibits a $(2 \times 1)$ reconstruction and all the deposited boron atoms are incorporated with the substrate. It is also important to determine where the boron atom are located, on or below the surface. To do this, we compare the spectrum of the $B / \operatorname{Si}(100)$ with those of the well studied $B / \operatorname{Si}(111)-(\sqrt{3} \times \sqrt{3})$ surface, where $B$ occupies a sublayer $S_{5}$ site. We find that the two surface are distinctively different. First, the ratio between B-related component and the bulk component (R/B) for the $\mathrm{B} / \mathrm{Si}(100)$ is much less than one, while this ratio is close to two for the $\mathrm{B} / \mathrm{Si}(111)$ surface [11-13]. It has been confirmed that the large $\mathrm{R} / \mathrm{B}$ ratio for the $\mathrm{B} / \mathrm{Si}(111)$ arises from the fact that B is located at the subsurface site and its capability to affect all the $\mathrm{Si}$ atoms that have direct bonding with it, which in this case is more than one monolayer (five Si atoms for every $\mathrm{B}$ atom) [11-13]. The lower R/B ratio observed for the $\mathrm{B} / \mathrm{Si}(100)$ surface indicates that much fewer $\mathrm{Si}$ atoms directly bond to $\mathrm{B}$, which further suggests that $B$ resides on the top surface. Another significant difference between the two surfaces is the response of the Si $2 p$ core level spectrum to further change of the surface environment induced by deposition of adsorbates. In case of the $\mathrm{B} / \mathrm{Si}(111)$ structure, it is extremely inert to change of the outside environment. The interface remains practically undisturbed and the Si 2 p spectrum is, to large extent, unchanged with or without adsorbates $[5,11]$. For the $\mathrm{B} / \mathrm{Si}(100)$, the situation is completely different. The Si $2 \mathrm{p}$ lineshape is very sensitive to the presence of other adsorbates, a situation much similar to that of the clean $\mathrm{Si}$ surface. Both of these differences strongly suggest that $B$ is located on the top surface instead of the subsurface.

Figure 3 presents the angle resolved valence band spectra $(\mathrm{hv}=21 \mathrm{eV})$ of the clean and Bcovered $\mathrm{Si}(100)$ surface at a normal emission configuration. The valence band spectrum of the $\mathrm{Ga} / \mathrm{Si}(100)$ surface is also included for sake of comparison. Several surface related 
states are highlighted by the tic-marks. The valence band spectrum of the $\mathrm{B} / \mathrm{Si}(100)-(2 \times$ 1) is found to be analogous to that of the clean $\mathrm{Si}(100)$ surface, though we do not see any sharp feature for the $\mathrm{B} / \mathrm{Si}(100)$ surface. It is particularly worth mentioning that the feature near the Fermi level is still present for this surface with reduced intensity. This feature represents the dangling bond states of the surface. It is in sharp contrast to the $\mathrm{Ga} / \mathrm{Si}(100)$ $(2 \times 1)$ surface, where one does not see any emission near the Fermi level. We attribute such drastic difference to presence of the dangling bonds at the $\mathrm{B} / \mathrm{Si}(100)$ surface, while all the dangling bonds are saturated at the $\mathrm{Ga} / \mathrm{Si}(100)$ surface.

The experimental results can be briefly summarized as the follows. We have found the $1 / 2$ ML of $\mathrm{B}$ on the $\mathrm{Si}(100)$ surface gives rise to a $(2 \times 1)$ surface reconstruction. But the surface is not a dangling bond free surface. Based on these results, we propose two possible structural models for this surface, and the schematic diagrams are presented in figure 4. In model (a) B is located at trench site between the two original dimers, while in model (b) B is located directly on top of the second layer Si atoms. Strickly speaking, model (a) represents the true $(2 \times 1)$ reconstruction, while model (b) will show a $(1 \times 2)$ LEED pattern. Since the substrate used here has two domain $(2 \times 1)$ reconstruction to start with, both $(2 \times 1)$ and $(1 \times 2)$ reconstructions will show the same LEED pattern. At the current stage, we have not performed calculation to determine which one has lower total energy. The existing calculation does not seem to give a definite answer either. Certain facts have to be taken into account in the calculation. Due to extremely small covalent radius of the $\mathrm{B}$ atom $(0.82 \AA)$, the short bond length of the Si-B bond may poses significant amount of strain in configuration (a). While one does not have such a concern for configuration (b), it presents strong distortion of the bond angle originated from the existing Si-dimers. Despite these difference, the two models possess some common properties. In both models, B acts as surface adatoms while the substrate structure is 
largely unchanged, including the original surface dimers. Furthermore, the number of the surface dangling bonds is reduced by a amount of at least $50 \%$, which can lower the total energy of the system. More reduction may result from possible formation of the bond between $\mathrm{B}$ and the Si right underneath in configuration (b). However, it is likely that the surface dangling bonds have not been completely eliminated. This appears to be consistent with the valence band spectrum shown in figure 2 .

It is interesting to compare the $\mathrm{B} / \mathrm{Si}(100)$ surface with the other group-III/Si(100) surfaces. As mentioned before, other group-III/Si(100) surfaces show a $(2 \times 2)$ surface reconstruction at a coverage of $1 / 2 \mathrm{ML}$. Structural models have been proposed for these surfaces. The current consensus is that these metal atoms are located on the top surface and form ad-dimers, while the substrate surface structure remains much the same $[14,15]$. The question regarding whether the ad-dimer is orthogonal or parallel to the original surface dimer remains to be answered. Nevertherless, these surfaces are dangling bond free since all the original surface dangling bonds and the bonds of the trivalent metal atoms are saturated, resulting from ad-dimer formation. Consequently, one dose not expect state near the surface Fermi level. That is what exactly we have observed. One reason why B does not form ad-dimer certainly originates from the much short B covalent radius compare with $\mathrm{Al}, \mathrm{Ga}$, and In (the covalent radii of these atoms are $1.18,1.26$, and $1.44 \AA$, respectively, while that of $\mathrm{Si}$ is $1.11 \AA$ ).

Finally, we have grown $\mathrm{Si}$ and $\mathrm{Ge}$ on the $\mathrm{B} / \mathrm{Si}(100)-(2 \times 1)$ structures. It is found that epitaxial Si and Ge layer could be grown on this structure with good epitaxy at relatively low temperature temperature $\left(<500^{\circ} \mathrm{C}\right)$. It is true with or without assistance from Sb as surfactant [16]. Homoepitaxial growth on this structure has been reported by Heatrick et al [17]. Essentially there is no much difference between the clean and B-covered $\mathrm{Si}(100)$ surface in terms of the epitaxial growth. On the contrary, high quality epitaxial growth on -7 . 
the $\mathrm{B} / \mathrm{Si}(111)$ surface can be relatively difficult, particularly at low temperature $\left(<400^{\circ} \mathrm{C}\right)$. Islanding in the overlayer may occur during growth because the surface energy of clean $\mathrm{Si}$ is much higher than that of the $\mathrm{B} / \mathrm{Si}(111)$ surface.

One of the most important properties the $\mathrm{B} / \mathrm{Si}(100)$ structure has is its capability to form a confined high doping area, the so-called delta doping. It has been discovered that all the $1 / 2 \mathrm{ML}$ boron atoms become electrically active underneath the epitaxially grown Si layers determined by an electrical measurement [17]. The spreading of this doping layer is also extremely small ( $<3$ layer), so that a nearly ideal delta doping layer is formed. We, in this work, use core level photoemission to determine the surface Fermi level position. Figure 5 shows the results of various surfaces with different structures. Essentially, we find that when our $\mathrm{B} / \mathrm{Si}(100)$ surface is covered by an epitaxial Si layer, the surface Fermi level continuous moving towards the valence band maximum. It indicates that there exists a ptype region near the surface for this n-type wafer. This result is clear consistent with the assumption that boron atoms underneath the epitaxial layer act like acceptors, and it strongly supports the previous finding from a microscopic point of view.

\section{Conclusions}

We have studied the atomic and electronic structure of the $\mathrm{B} / \mathrm{Si}(111)-(2 \times 1)$ surface prepared by $\mathrm{B}_{2} \mathrm{O}_{3}$ deposition and subsequent high temperature annealing. Our results show that the $\mathrm{B}$, in this case, is located on the top surface as adatom. With the structure models we have proposed, there is a reduction of the surface dangling bond, although the surface dangling bonds are not completely saturated. Epitaxial growth of Si and Ge layer on top of this surface has been achieved at relatively low temperatures. Upon the growth, there is a type conversion near the surface region. The results indicate that boron atoms 
now act as acceptors, supporting previous finding of formation of B delta doping layers under the similar conditions.

\section{Acknowledgment}

The work was done at SSRL that is operated by the Department of Energy, Division of Chemical Sciences. The authors also acknowledge the support of the office's Division of Material Science for this research. One of the authors (RC) thanks J.E. Northrup for helpful discussion.

\section{References}

1. I.W. Lyo, E. Kaxiras, and Ph. Avouris, Phys. Rev. Lett. 63, 1261 (1989).

2. P. Bedrossian, R.D. Meade, K. Mortensen, D.M. Chen, J.A. Golovchenko, and D. Vanderbilt, Phys. Rev. Lett. 63, 1257 (1989).

3. R.L. Headrick, I.K. Robinson, E. Vlieg, and L.C. Feldman, Phys. Rev. Lett. 63, 1253 (1989).

4. I.-W Lyo and Ph. Avouris, Science 245, 1369 (1989).

5. Ph. Avouris, J. Phys. Chem. 94, 2246 (1990).

6. A.A. Baski, J. Nogami, and C.F. Quate, J. Vac. Sci, Technol. A 8, 245 (1990); J. Vac. Sci. Technol. A. 9, 1946 (1991); Phys. Rev. B 43, 9316 (1991); and Phys. Rev. B 44, 1415 (1991).

7. R.L. Headrick, B.E. Weir, A.F.J. Levi, D.J. Eaglesham, and L.C. Feldman, Appl. Phys. Lett. 57, 2779 (1990).

8. B.E. Weir, R.L. Headrick, Q. Shen, L.C. Feldman, M.S. Hybertsen, M. Needels, M. Schlüter, and T.R. Hart, Phys. Rev. B 46, 12861 (1992). 
9. R. Cao, X. Yang, J. Terry, and P. Pianetta, Phys. Rev. B 45,13749 (1992).

10. D.-S. Lin, T. Miller, and T.-C. Chiang, Phys. Rev. Lett. 67, 2187 (1991).

11. R. Cao, X. Yang, and P. Pianetta, (to be published).

12. A.B. McLean, L.J. Terminello, and F.J. Himpsel, Phys. Rev. B 41, 7694 (1990).

13 J.E. Rowe, G.K. Wertheim, and D.M. Riffe, J. Vac. Sci. Technol. A 9, 1020 (1991).

14. B. Bourguignon, K.L. Carleton, and S.R. Leone, Surf. Sci. 204, 455 (1988).

15. J.E. Northrup, M.C. Schabel, C.J. Karlsson, and R.I.G. Uhrberg, Phys Rev. B 44, 13799, (1991).

16. R. Cao, X. Yang, J. Terry, and P. Pianetta, Appl. Phys. Lett. 61, 2347 (1992).

17. R.L. Headrick, B.E. Weir, A.F.J. Levi, B. Freer, J. Bevk, and L.C. Feldman, J. Vac. Sci. Technol. A 9, 2269 (1991). 


\section{Figure Captions}

Figure 1. The B 1 s core level spectra taken at $\mathrm{hv}=220 \mathrm{eV}$ as a function of annealing temperatures.

Figure 2. The Si $2 p$ core level spectra, taken at $h v=150 \mathrm{eV}$, of the clean and Bcovered $\mathrm{Si}(100)-(2 \times 1)$ surface. The spectra have been decomposed into different components. Note that for the $\mathrm{B} / \mathrm{Si}(100)$ surface, $\mathrm{S}$ is replaced by $\mathrm{S}^{*}$, a B-related chemically shifted Si component.

Figure 3. The angle revolved valence band spectra as a function of photoelectron emission angle (incident angle $45^{\circ}$ ), taken at $\mathrm{hv}=21 \mathrm{eV}$, of the $\mathrm{Si}(100), \mathrm{B} / \mathrm{Si}(100)$, and $\mathrm{Ga} / \mathrm{Si}(100)$ surfaces.

Figure 4. The schematic model of the $\mathrm{B} / \mathrm{Si}(100)$ surface. Boron atoms reside on the trench adatom sites in model (a), showing a $(2 \times 1)$ reconstruction, while they are located on the top of the second layer Si atoms in model $(b)$, showing a $(1 \times 2)$ reconstruction.

Figure 5. The surface Fermi level positions determined from Si $2 \mathrm{p}$ core level spectra for various surfaces. Notice that there is a further movement of the surface Fermi level toward the valence band maximum, indicating formation of p-type region near the surface upon Si epitaxial growth on the $\mathrm{B} / \mathrm{Si}(100)$ structure. 


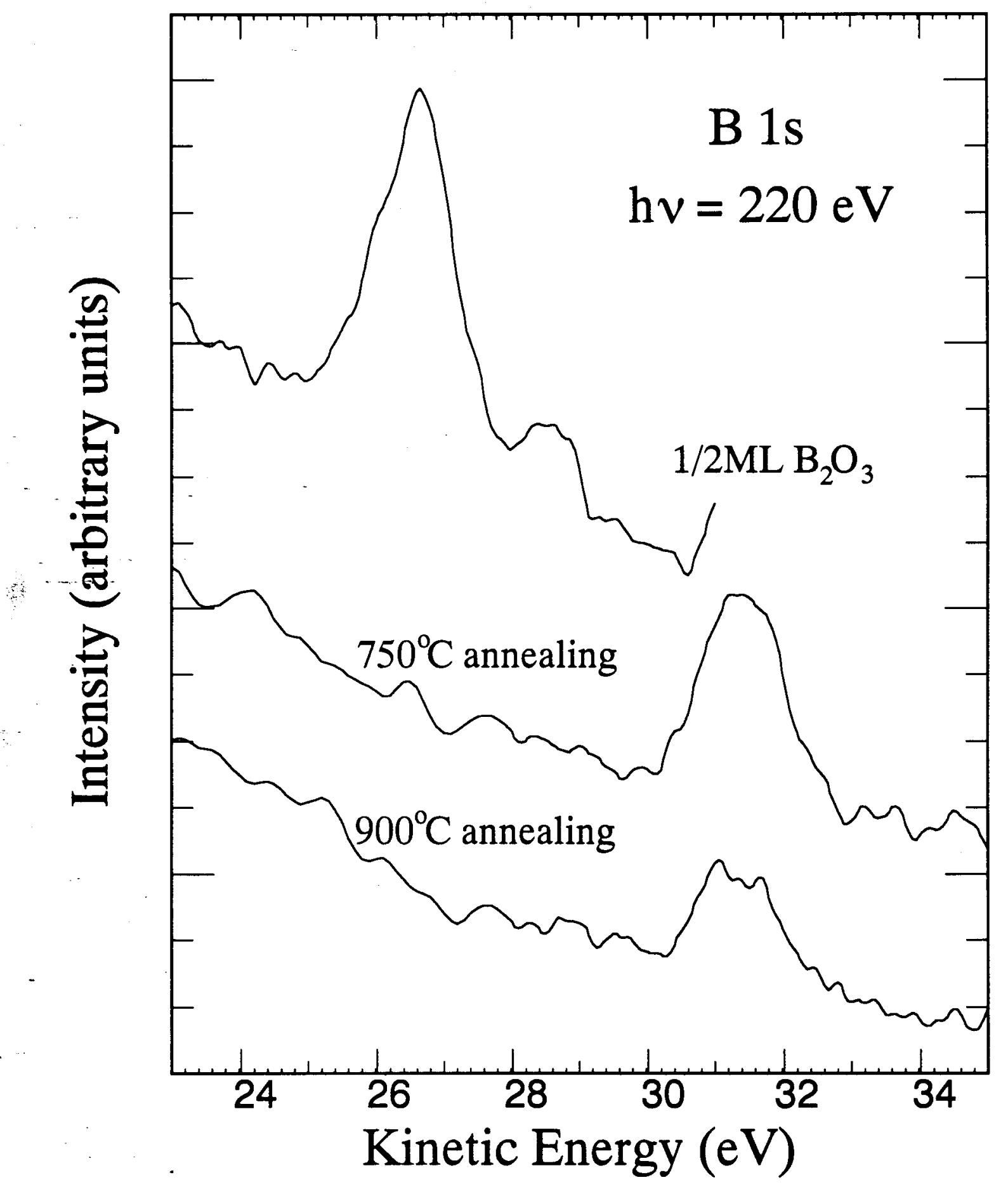

Fig. 1 


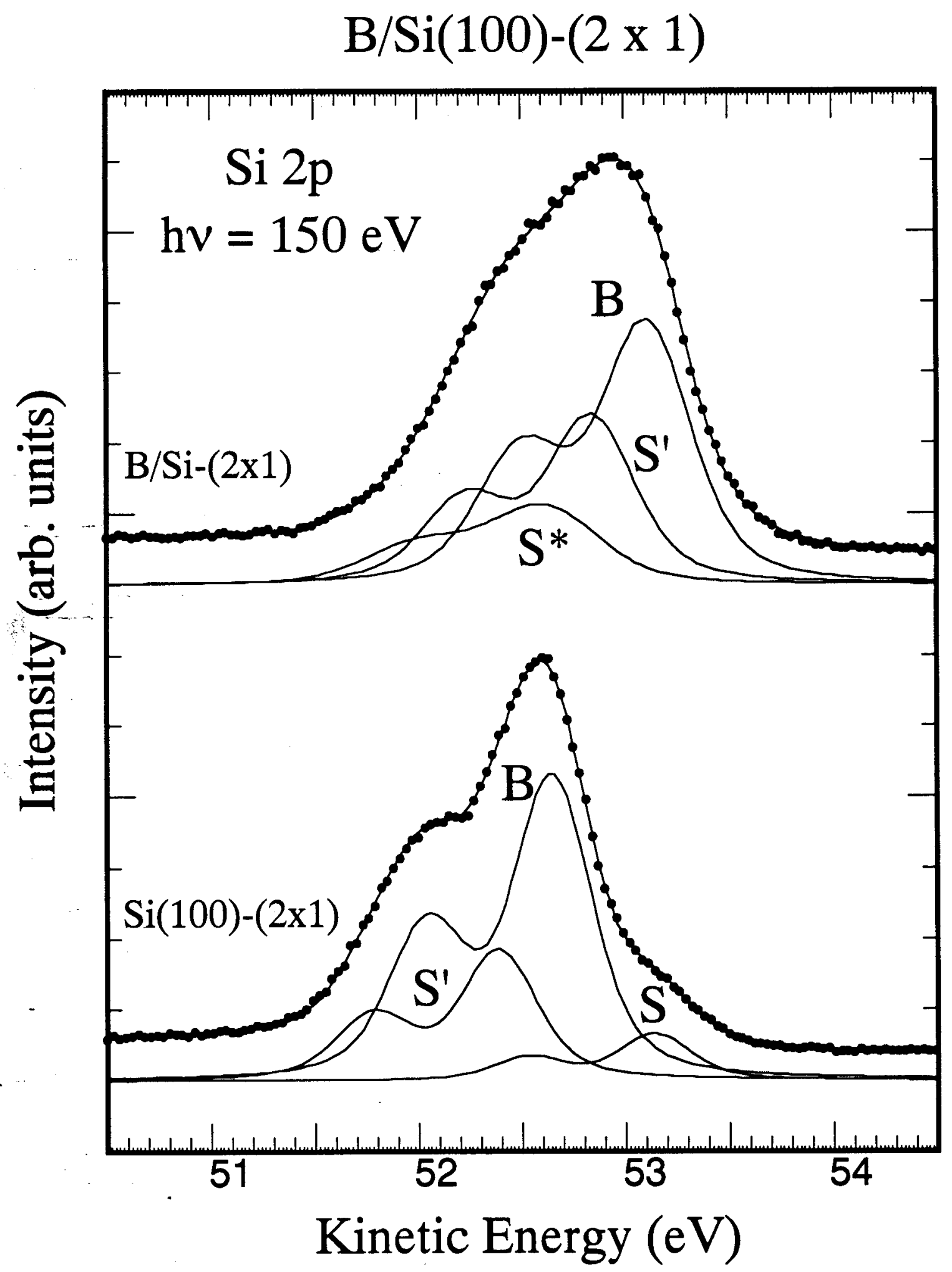

Fig 2 


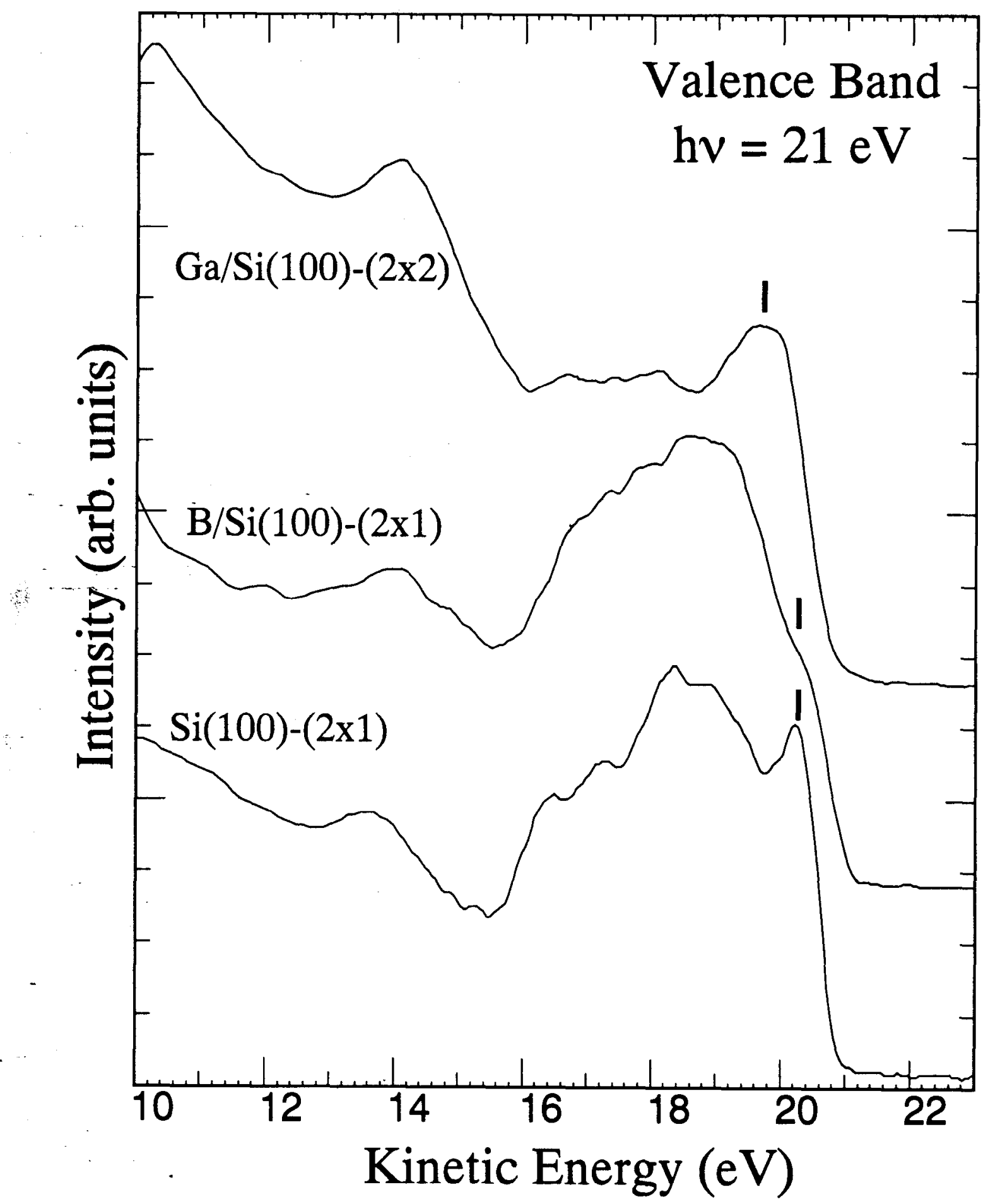

Fig. 3 
(a) $2 \times 1$ Trench adatom

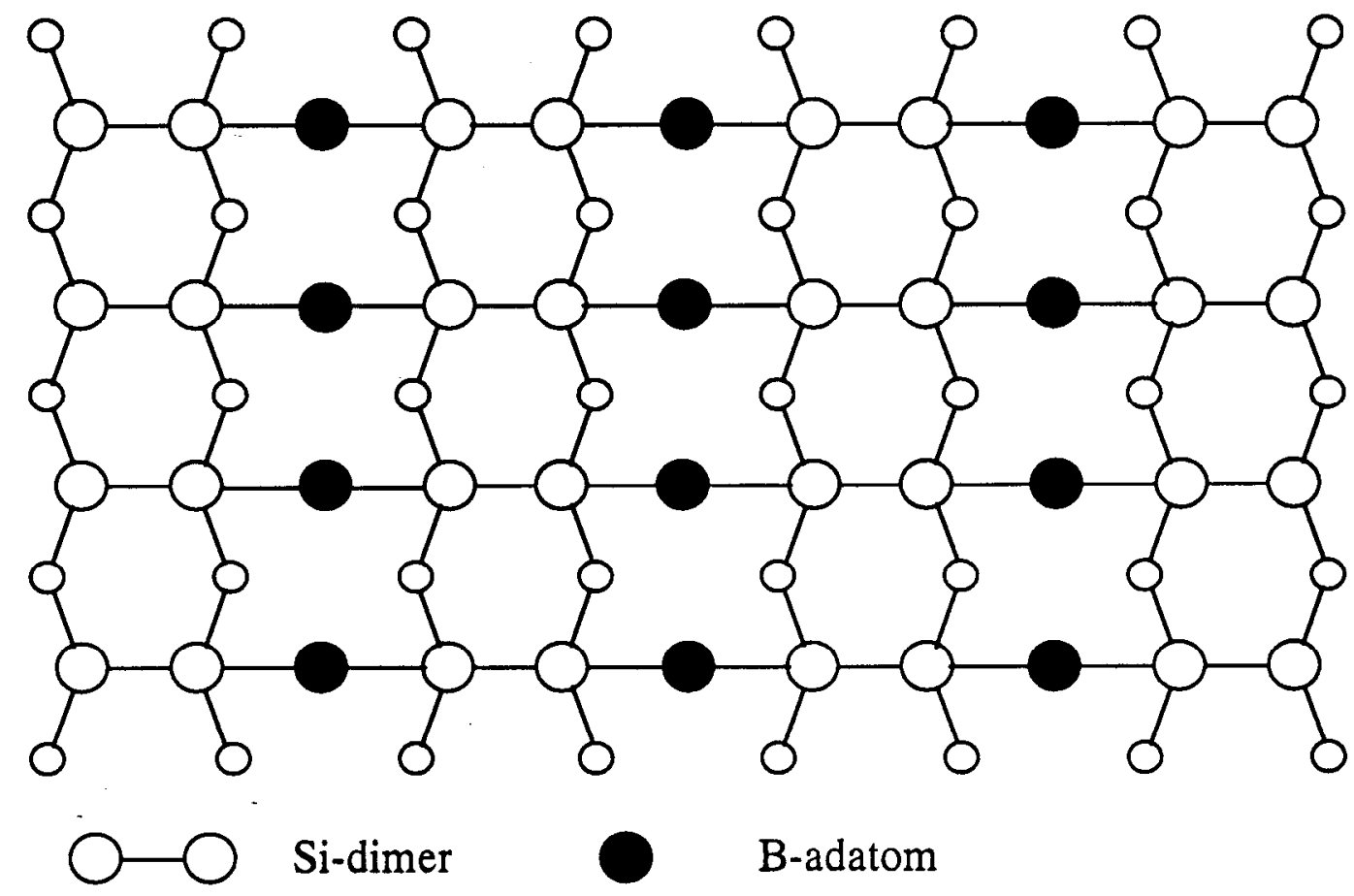

(b) $1 \times 2 T_{3}$ adatom

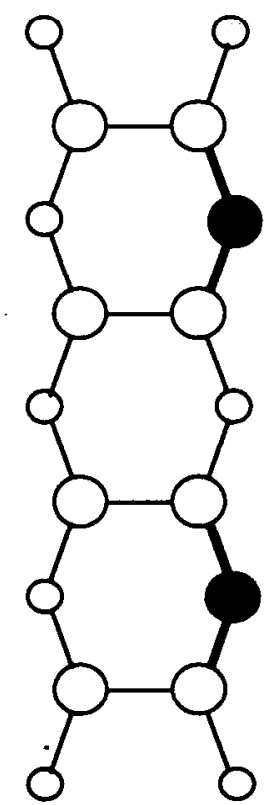

$\bigcirc-\bigcirc$ Si-dimer

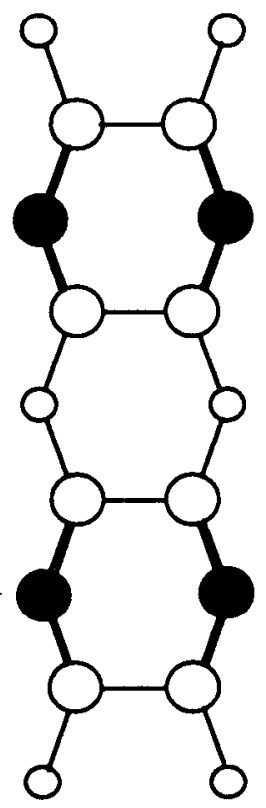

B-adatom
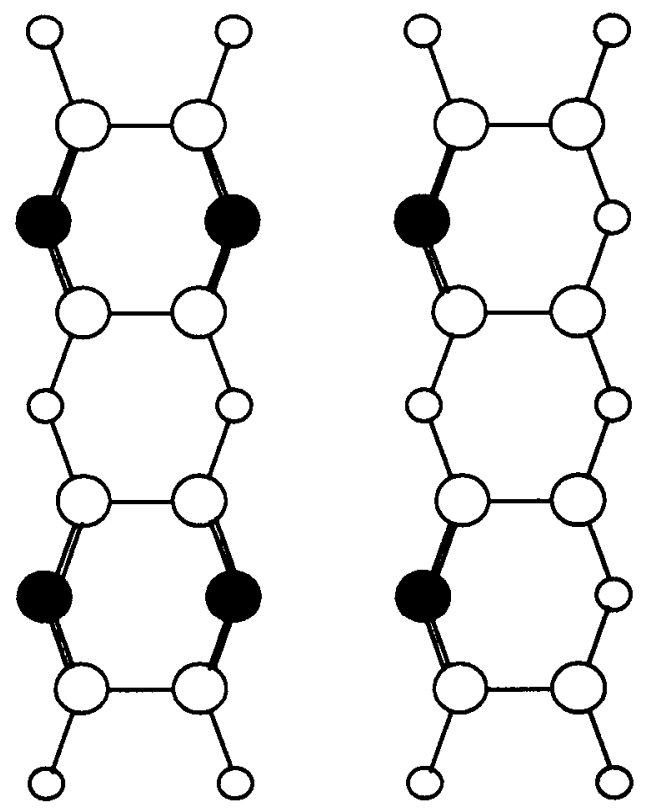

Fig. 4 


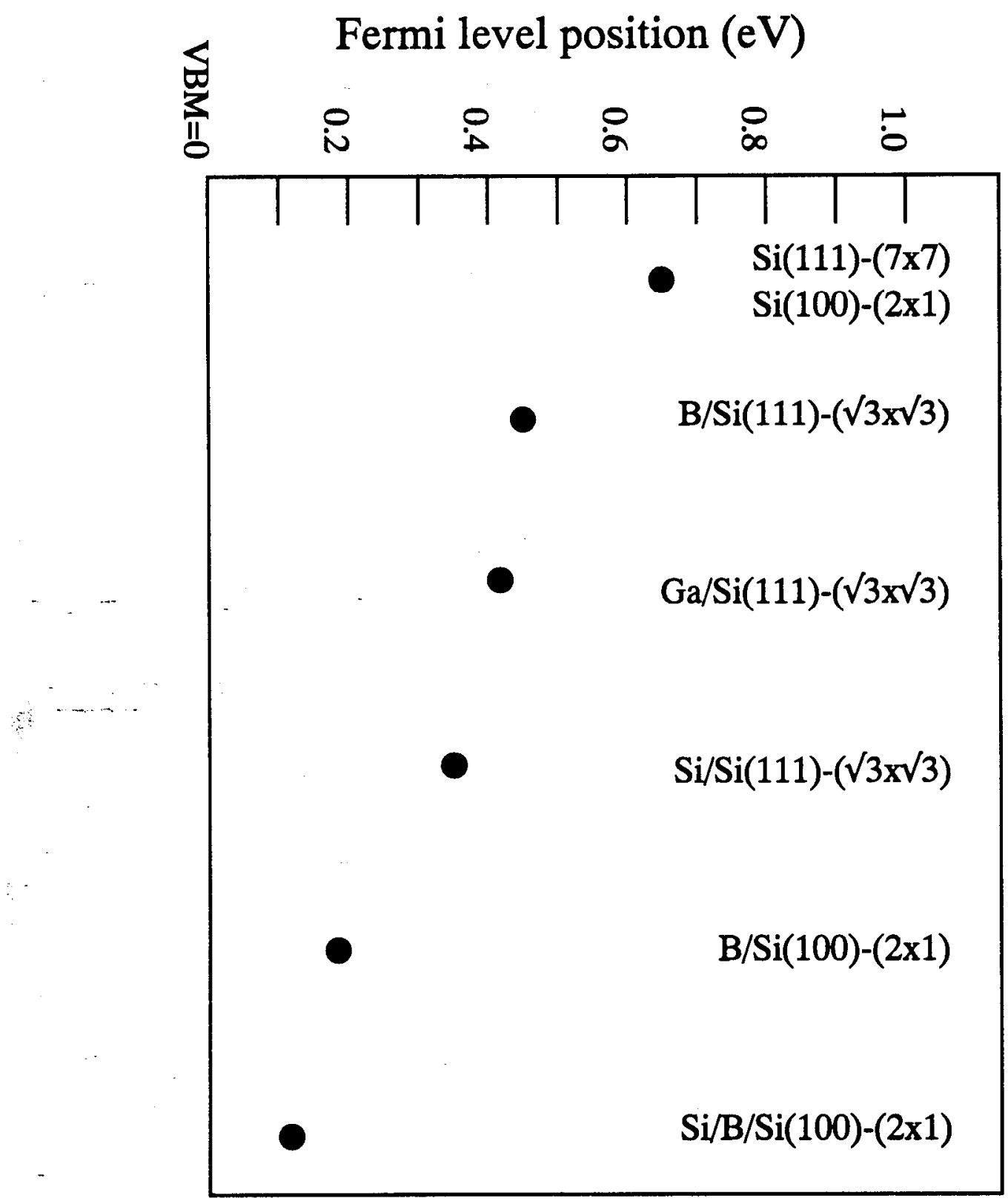

Fig. 5 